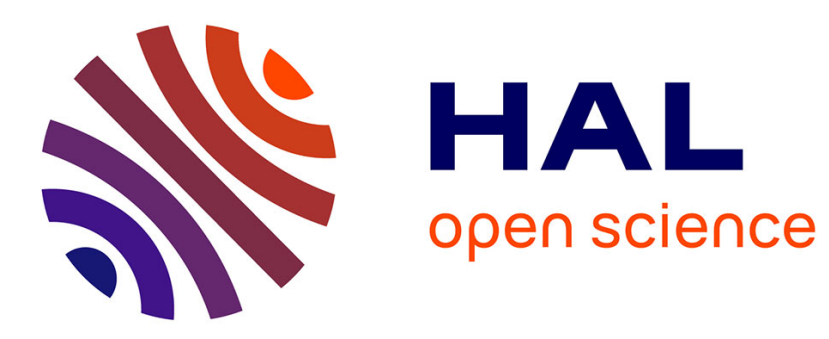

\title{
Numerical modeling of hydraulic fracture problem in permeable medium using cohesive zone model
}

\author{
Benoît Carrier, Sylvie Granet
}

\section{To cite this version:}

Benoît Carrier, Sylvie Granet. Numerical modeling of hydraulic fracture problem in permeable medium using cohesive zone model. Engineering Fracture Mechanics, 2012, 79, pp.312-328. 10.1016/j.engfracmech.2011.11.012 . hal-00670787

\section{HAL Id: hal-00670787 \\ https://hal.science/hal-00670787}

Submitted on 16 Feb 2012

HAL is a multi-disciplinary open access archive for the deposit and dissemination of scientific research documents, whether they are published or not. The documents may come from teaching and research institutions in France or abroad, or from public or private research centers.
L'archive ouverte pluridisciplinaire HAL, est destinée au dépôt et à la diffusion de documents scientifiques de niveau recherche, publiés ou non, émanant des établissements d'enseignement et de recherche français ou étrangers, des laboratoires publics ou privés. 


\title{
Numerical modeling of hydraulic fracture problem in permeable medium using cohesive zone model
}

\author{
Benoit Carrier ${ }^{\mathrm{a}, \mathrm{b}, *}$, Sylvie Granet ${ }^{\mathrm{b}}$ \\ ${ }^{a}$ Université Paris-Est, Laboratoire Navier (ENPC/IFSTTAR/CNRS), École des Ponts \\ ParisTech, 6-8 avenue Blaise Pascal, 77455 Marne-la-Vallée, France \\ ${ }^{b}$ Laboratoire LaMSID, UMR EDF-CNRS, 1 avenue du Général de Gaulle, 92140 \\ Clamart, France
}

\begin{abstract}
This paper considers the problem of a fluid-driven fracture propagating in a permeable poroelastic medium. We develop a zero-thickness finite element to model the fracture. The fracture propagation is governed by a cohesive zone model and the flow within the fracture by the lubrication equation. The hydro-mechanical equations are solved with a fully coupled approach, using the developed zero-thickness element for the propagating fracture and conventional bulk finite elements for the surrounding medium. The numerical results are compared to analytical asymptotic solutions under zero fluid lag assumption in the four following limiting propagation regimes: toughnessfracture storage, toughness-leak-off, viscosity-fracture storage and viscosityleak-off dominated. We demonstrate the ability of our cohesive zone model in simulating the hydraulic fracture in all these propagation regimes.
\end{abstract}

Keywords: Hydraulic fracture, Cohesive zone model, Finite element analysis, Hydro-mechanical coupling

\section{Introduction}

The propagation of fluid-driven fractures in a porous medium is an important problem in rock mechanics. Because these discontinuities affect the hydro-mechanical properties of rock formations, they are of special interest in many engineering fields. Hydraulic fracturing is a commonly used technique in petroleum engineering to enhance reservoirs permeability and wells efficiency. Other applications of fluid-driven crack problem include underground storage of carbon dioxide, toxic or radioactive waste [30], geothermal

\footnotetext{
*Corresponding author

Email address: benoit.carrier@enpc.fr (Benoit Carrier)
} 
energy production stimulation [48] and geophysics, for example water-driven propagation of fracture along glacier beds [44].

Fluid-driven fractures were studied by many contributors during the last decades from an analytical perspective $[3,7,14,21,35,34,41,25,27,22,38$, $36]$ as well as from a numerical point of view [10, 23, 29, 31, 42, 43]. However, because of the strongly non-linear, non-local and history-dependent response and because of the tip singularity, modeling hydraulic fractures is a challenging problem. Hydraulic fracturing involves several coupled phenomena [3]:

- the preferential fluid flow in the fracture depends on the fracture aperture;

- mechanical deformation of the surrounding porous medium is induced by the fluid pressure on fracture lips;

- the fracture and the porous medium exchange fluid;

- the fracture propagates, and therefore, hydraulic fracture is a moving boundary problem.

A system of non-linear partial differential equations can be associated to this list of coupled processes. During the last sixty years, numerous papers $[3,7,14,21,35,34,41,25,27,22,38,36,1]$ addressed the mathematical formulation of the hydraulic fracture problem and the search for analytical solutions. These works focused on the injection of an incompressible Newtonian fluid in an elastic impermeable or permeable medium for simple fracture geometries: plane strain KGD [27, 22] or PKN [38, 36] models and axisymmetric penny-shaped model [1]. Asymptotic solutions were derived for these idealized models. The analysis tool they constitute can be used as a benchmark for numerical simulations. In the recent years, a scaling and asymptotic framework was built to determine the influence of the physical processes involved in the fracture propagation $[14,35]$. Two competing asymptotic dissipation mechanisms and two competing storage mechanisms were highlighted. Energy is dissipated by fracture propagation and by fluid viscous flow in the fracture. Fluid storage processes are fluid leak-off in the porous solid and fluid storage in the crack. To these two sets of mechanisms are associated the four following combined asymptotic regimes: storage-toughness [21], storage-viscosity [41], leak-off-toughness [7] and leak-off-viscosity [3].

To deal with more complex fracture geometries, numerical tools were developed to simulate the hydraulic fracture problem [2]. For instance, various 
finite element-based methods were applied to the simulation of discrete fluiddriven fracture. Simoni and Secchi [43] used a re-meshing algorithm and a staggered solving algorithm to model cohesive fracture propagation under fluid pressure. They considered fluid exchanges between the fracture and the porous medium. Lecampion [29] investigated the extended finite element method to model hydraulic fracture in an impermeable medium. He proposed the use of special enrichment functions at the fracture tip to capture the aperture and pressure singularities. However, neither did he considered the longitudinal fluid flow nor the fracture propagation. Another set of methods is based on zero-thickness interface elements. In their pioneering work, Boone and Ingraffea [6] used the cohesive zone model to model fracture propagation in a poroelastic medium. The problem was solved by finite element method for the poroelastic medium, along with a finite difference method for the fluid flow in the fracture. Segura and Carol [42] and Guiducci et al. [23] developed respectively a double-noded and a triple-noded zero-thickness finite element. They modeled longitudinal and transversal flow in preexisting discontinuities. Chen et al. [10] adopted an interface element governed by a cohesive law to model fracture propagation in an impermeable medium. The agreement of their numerical results with the toughness-dominated solution was very good. Lobao et al. [31] modeled both fluid leak-off and propagation with a zero-thickness element. They investigated the effect of rock plasticity, as Papanastasiou [37] did for impermeable medium. In some recent contributions, Sarris [40, 39] examined the influence of the fracture process zone and of the permeability of the surrounding medium. However, building a robust and accurate three-dimensional numerical method taking into account all the phenomena listed above still remains a challenging task.

In this work, we describe a numerical method to model the hydro-mechanical evolution both of the fracture and of the surrounding rock in the finite element analysis framework. For this purpose, a zero-thickness element is developed to model the discontinuity. We consider both the propagation and the exchanges of fluid with the permeable porous medium. Fracture propagation is governed by a cohesive law. The fluid pressure in the fracture acts as a mechanical and hydraulic boundary condition on the poroelastic surrounding medium. We compare the results of our model with analytical solutions and check the validity of our model in all asymptotic propagation regimes. We especially investigate the ability of cohesive elements to model fluid-driven crack propagation in the viscosity-dominated regime. Indeed, hydraulic fractures for reservoir stimulation typically propagate in the viscosity-dominated regime [17]. Therefore, this propagation regime is the focus of numerous works $[3,41]$. We also analyze the influence of the permeability of the surrounding medium on the fluid diffusion pattern in the 
rock and the associated poroelastic response on fluid-driven fracture propagation. The classic assumption of one-dimensional diffusion (Carter model [8]) around the fracture is no longer valid in the case of water flooding technique for instance [28] and three-dimensional diffusion have to be considered [33]. Moreover, the pore pressure increases around the fracture and induces a back-stress which tends to shut the fracture [15, 45, 28]. We investigate the extent of this poroelastic effect.

In section 2, we focus on the governing equations of the coupled problem: lubrication equation, pressure continuity and cohesive zone model. Section 3 is devoted to a detailed description of the zero-thickness finite element and of the FEM formulation of the problem. In section 4, our model is applied to the KGD plane-strain model and its results are presented in the light of the relevant asymptotic framework. Finally, some concluding remarks are given in section 5 .

\section{Governing equations of the hydraulic fracture in a permeable medium}

\subsection{Fluid flow in the fracture and in the porous medium}

The longitudinal fluid flow in the fracture (figure 1-a) is described by the fluid mass conservation equation

$$
\frac{\partial \rho w}{\partial t}+\frac{\partial W}{\partial s}=0
$$

where $w$ is the local fracture width, $\rho$ is the fluid density, $W$ is the mass flow rate and $s$ is the longitudinal coordinate along the fracture $\Gamma$.

The seepage from the fracture to the surrounding porous medium is governed by the continuity of the pressure across the discontinuity (figure 1-b)

$$
p_{\mid \Gamma}^{+}=p_{f}=p_{\mid \Gamma}^{-}
$$

where $p_{f}$ is the pressure in the fracture and $p^{+}$and $p^{-}$are the pressures in the upper $(+)$ and lower $(-)$ surrounding medium, respectively.

The longitudinal flow is assumed to be coupled to the fracture aperture according to the local cubic law [46]

$$
W(s, t)=-\frac{\rho w(s, t)^{3}}{12 \mu}\left(\frac{\partial p_{f}}{\partial s}-\rho g \frac{\partial z}{\partial s}\right) .
$$

where $\mu$ is the fluid viscosity, $g$ the gravity acceleration and $z$ the local altitude. 
The cubic law is derived from Poiseuille equation between two smooth parallel plates. It was confirmed experimentally [46] and can be corrected to take into account surface roughness [32].

Neglecting gravity, we obtain the following lubrication equation

$$
\frac{\partial \rho w}{\partial t}-\frac{\partial}{\partial s}\left(\frac{\rho w^{3}}{12 \mu} \frac{\partial p_{f}}{\partial s}\right)=0 .
$$

At the crack tip, under the assumption of zero fluid-lag, the flow rate is equal to zero

$$
W(s=l, t)=0 .
$$

where $l$ is the fracture length.

$\mathbf{a}$

A

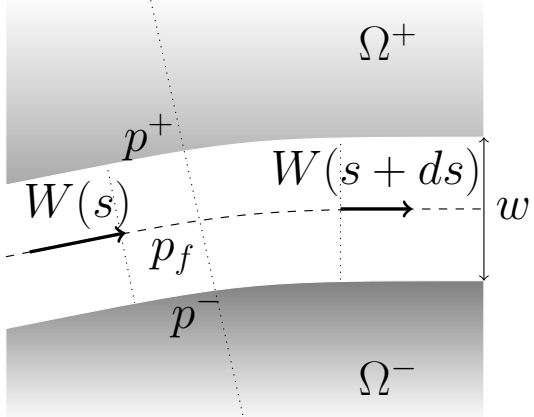

$\mathrm{A}^{\prime}$ b

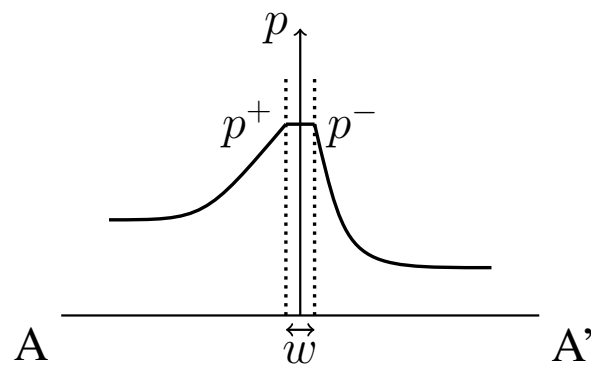

Figure 1: Fluid flow modeling in the broken part of the fracture (a) and pressure around the fracture (b).

The behavior of the surrounding saturated porous medium is governed by the hydro-mechanical equations of poromechanics [12]:

$$
\begin{gathered}
\operatorname{Div}\left(\boldsymbol{\sigma}^{\prime}-b \mathbf{I d}\right)=\mathbf{0} \\
\frac{\partial}{\partial t} \rho \Phi=\rho_{0} \frac{\kappa}{\mu} \Delta p
\end{gathered}
$$

and

$$
\Phi-\Phi_{0}=b \varepsilon_{V}-\left(\frac{1}{M}-\frac{\Phi_{0}}{K_{f}}\right) p
$$

where $\boldsymbol{\sigma}^{\prime}$ is the Biot effective stress $\left(\boldsymbol{\sigma}^{\prime}=\boldsymbol{\sigma}+b \mathbf{I d}\right), b$ is the Biot coefficient, $\Phi$ and $\Phi_{0}$ are the Lagrangian porosity in the actual configuration and in the 
reference configuration, respectively, $\kappa$ is the intrinsic permeability, $\varepsilon_{V}$ is the volumetric strain, $M$ the Biot modulus and $K_{f}$ the pore fluid compressibility.

The conditions of continuity across the discontinuity are the equation of continuity of fluid pressure (equation (2)), the continuity of stress

$$
\boldsymbol{\sigma} \cdot \mathbf{n}=p_{f},
$$

and the equality of fracture aperture and normal displacement jump

$$
d w=d \llbracket \mathbf{u} . \mathbf{n} \rrbracket
$$

where $\mathbf{n}$ is the local normal, and $\mathbf{u}$ the displacement field in the porous medium.

\subsection{Fracture Propagation}

In this work, we assume that fracture propagation is governed by a cohesive zone model. This model was proposed by Dugdale [16] and Barenblatt [4] to overcome the Griffith brittle fracture theory defects. In particular, it avoids the stress singularity at the fracture tip. It is based on Khristianovitch hypothesis [47]: the loading on fracture lips always has to ensure the finiteness of stress at the fracture tip. The existence of a cohesive zone is postulated at the fracture tip, where forces are exerted on the emergent fracture lips. These cohesive forces act against the opening forces and compensate the stress singularity at the tip. We only consider the class of solutions correspoding to the case of a zero stress intensity factor. The process zone undergoes plastic deformation and micro-cracking under the tension forces and acts as a transition zone between the open fracture and the undamaged material. The cohesive zone framework has been successfully applied to model fracture in ductile metals and polymers [19], cement-based materials [24] and geomaterials. In the case of hydraulic fracture, the pressure can also be singular at the fracture tip in the Griffith theory framework [13]. With the cohesive zone model, the fracture tip is not sharp and the pressure singularity can be removed.

The behavior of the cohesive fracture is given by a cohesive law, which relates the tension across the interface $\boldsymbol{\tau}$ to the displacement jump $\boldsymbol{\delta}$ and to the cohesive energy density $\Psi$ :

$$
\boldsymbol{\tau}=\frac{\partial \Psi}{\partial \boldsymbol{\delta}}
$$

Since our fracture is invaded by a liquid at pressure $p_{f}$, the lips of the broken part of the fracture are not stress-free. To take into account the effect 
of the pressure, and considering the matrix is plastically incompressible [12], we introduce the effective stress $\boldsymbol{\tau}^{\prime}$. The relationship between total stress and effective stress across the discontinuity is

$$
\boldsymbol{\tau}=\boldsymbol{\tau}^{\prime}-p_{f} \mathbf{n}
$$

Thus, similarly to bulk poromechanics, the cohesive law actually relates $\boldsymbol{\tau}^{\prime}$ to $\boldsymbol{\delta}$.

This cohesive energy density must take into account perfect adhesion before fracturation, zero cohesive force when the fracture is open, fracturation irreversibility and non-interpenetration of fracture lips. Here, we use a linear cohesive law, displayed in figure 2. Since the fluid pressure in the crack induces only normal forces, we focus on mode-I propagation.

$\mathbf{a}$

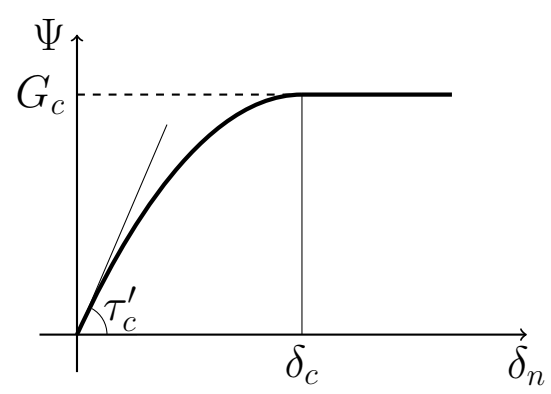

b

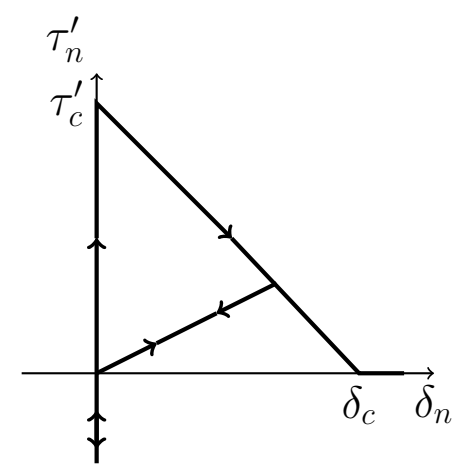

Figure 2: Quadratic cohesive energy density (a) and corresponding linear cohesive law in the direction normal to the fracture (b).

There is a perfect bonding until the tension reaches the critical stress $\tau_{c}^{\prime}[9]$. Then, the interface begins to be damaged. When the total fracture energy $G_{c}$ is dissipated, the fracture lips are stress-free.

Instead of the fracture toughness in mode-I $K_{I c}$ in Griffith theory, this cohesive law requires two independent material parameters: the cohesive energy $G_{c}$ and the critical stress $\tau_{c}^{\prime}$. The total separation width $\delta_{c}$ is given by the following relationship:

$$
G_{c}=\frac{1}{2} \tau_{c}^{\prime} \delta_{c}
$$

Figure 5 shows a representation of the cohesive zone.

If the cohesive zone is small compared to the fracture length, the fracture toughness and the cohesive energy are related by Irwin's formula [26]: 


$$
K_{I c}=\sqrt{G_{c} \frac{E}{1-\nu^{2}}}
$$

where $E$ is the Young's modulus and $\nu$ the Poisson's ratio.

\subsection{Regimes of propagation and available solutions}

The hypotheses of the analytical framework $[3,7]$ are similar to the ones of our model (lubrication equation, matrix elasticity). However, in the analytical model the propagation is governed by linear elastic fracture mechanics. Instead, we use a cohesive model. In the analytical model fluid leak-off is given by Carter's law [8] while our model solves the full equation of pressure diffusion.

Carter's model gives an expression for the leak-off from the fracture $g_{l o}(x, t)$

$$
g_{l o}(x, t)=\frac{2 C_{L}}{\sqrt{t-t_{0}(x)}}
$$

where $t_{0}(x)$ is the time at which the crack arrives at $x$ and $C_{L}$ is the leak-off coefficient. Carter's law can be derived from the pressure diffusion equation in an semi-infinite medium, assuming that the leak-off is unidimensional and that the hydraulic head $\Delta p=p_{f}-p_{0}$ is constant in time, where $p_{0}$ is the pore pressure. For hydraulic fractures, the constant hydraulic head assumption can be justified for high confinements [34]. In that case $p_{f} \approx \sigma_{0}$ and $\Delta p \approx \sigma_{0}-p_{0} \gg p_{f}-\sigma_{0}$, where $\sigma_{0}$ denotes the confining stress. The relationship between the leak-off coefficient and the parameters of the surrounding medium is given by

$$
C_{L} \approx \frac{\kappa}{\mu} \frac{\sigma_{0}}{\sqrt{\pi c}}
$$

where $c$ denotes the poroelastic intrinsic diffusion coefficient [12].

The hydraulic fracture propagation is governed by two competing energy dissipation mechanisms (viscous flow and fracturation) and two competing storage mechanisms (in the fracture or in the porous matrix). The relative magnitude of the dissipation processes on one hand and of the storage processes on the other hand can be described by a dimensionless toughness $\mathcal{K}$ and a dimensionless leak-off coefficient $\mathcal{C}$ [3], respectively:

$$
\mathcal{K}=\frac{4 K_{I c}}{\sqrt{\pi}}\left(\frac{1}{3 Q_{0} E^{\prime 3} \mu}\right)^{1 / 4} \quad \mathcal{C}=2 C_{L}\left(\frac{E^{\prime} t}{12 \mu Q_{0}^{3}}\right)^{1 / 6}
$$

where $E^{\prime}$ is the plane strain elastic modulus, $Q_{0}$ is the injection rate. 
Figure 3 shows the two-dimensional $(\mathcal{K}, \mathcal{C})$ parameter space. Each edge of this space represents an asymptotic regime. During the injection of a fluid in a plane-strain fracture, the propagation regime evolves from the storagedominated edge $(\mathcal{C} \ll 1)$ to the leak-off-dominated edge $(\mathcal{C} \gg 1)$ with time.

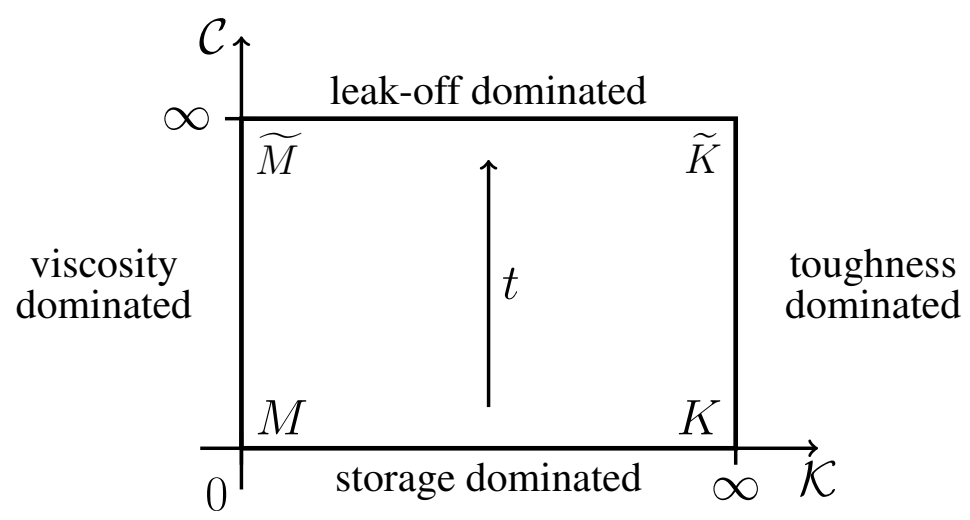

Figure 3: Hydraulic fracture parametric space (after [7]).

Chen et al. [10] showed the ability of a cohesive zero-thickness finite element to model the hydraulic fracture propagation in an impermeable medium in the toughness-dominated regime ( $K$ regime). In this work, we extend the use of a cohesive element to both the toughness-dominated ( $K$ and $\widetilde{K}$ regimes) and the viscosity-dominated ( $M$ and $\widetilde{M}$ regimes) propagations in a permeable medium. The fluid lag is always assumed to be equal to zero. This zero-lag assumption is valid if the confining stress is large enough [3].

We use the near- $K[7]$ and the near- $M[3]$ solutions, the small time solutions in the toughness and viscosity regimes respectively, and the the near- $\widetilde{K}$ $[7]$ and the near- $\widetilde{M}[3]$ solutions, the large time solutions in the toughness and viscosity regimes respectively.

\section{Finite element modeling of hydraulic fractures}

\subsection{Zero-thickness element}

The cohesive zone model can be easily implemented into classic finiteelement framework. Specific cohesive elements mesh the predicted fracture path $\Gamma$, while usual bulk elements mesh the neighboring medium $\Omega$. However, the fracture path must be postulated a priori. To model the fracture, we developed a specific zero-thickness cohesive element. This element is very similar to the zero-thickness elements previously used in mechanics [11]. However, it has additional degrees of freedom to take into account the 
hydro-mechanical coupling (see figure 4). This element is a degenerated 8node quadrangle. The fluid flow within the fracture is discretized on the mid-plane nodes (nodes 6 and 8 in figure 4). The outer segments are linked to the bulk hydro-mechanical elements. The fracture aperture is given by the displacement difference between the upper and the lower segments.

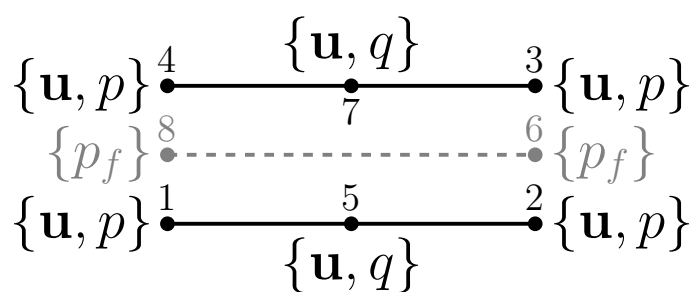

Figure 4: Zero-thickness interface element with hydro-mechanical coupling.

The quadratic cohesive energy $\Psi$ (figure 2) is not derivable in 0 with respect to $\delta_{n}$ and the cohesive traction - separation law has an infinite slope in 0 . Therefore, the cohesive energy is regularized near 0 and we give to the traction - separation a finite rigidity in 0 . We define the displacement jump $\kappa_{0}$ below which the cohesive law is elastic. When $\delta_{n} \geq \kappa_{0}$ the cohesive law has the softening behavior displayed in figure 2. In all the simulations, we took $\kappa_{0}=5.10^{-4} \times \delta_{c}$ and checked that this value had no influence on the results.

Consistently with the bulk hydro-mechanical elements we used, we choose bilinear interpolation functions for the displacement $\mathbf{u}$ and linear interpolation functions for the pressures $p$ and $p_{f}$. The choice of $\mathrm{P} 2 / \mathrm{P} 1$ interpolation for $\mathbf{u}-p$ formulations was shown by [20] to guarantee an optimal convergence of the error estimators in poroelasticity and to reduce the oscillations of the numerical solution when sudden boundary conditions are applied.

The discrete displacement field is approximated by

$$
\mathbf{u}(\mathbf{x}) \approx[\mathbf{N}(\mathbf{x})] .\{\mathbf{U}\}
$$

where $[\mathbf{N}(\mathbf{x})]$ is the matrix of quadratic interpolation functions and $\{\mathbf{U}\}$ is the displacement nodal vector. The discrete displacement on the upper and lower fracture lips $\Gamma^{+}$and $\Gamma^{-}$are interpolated by

$$
\mathbf{u}^{+}(\mathbf{s}) \approx\left[\mathbf{N}_{+}(\mathbf{s})\right] \cdot\{\mathbf{U}\} \text { and } \mathbf{u}^{-}(\mathbf{s}) \approx\left[\mathbf{N}_{-}(\mathbf{s})\right] .\{\mathbf{U}\}
$$

where $\left[\mathbf{N}_{+}(\mathbf{s})\right]$ and $\left[\mathbf{N}_{+}(\mathbf{s})\right]$ are the traces of $[\mathbf{N}(\mathbf{s})]$ on $\Gamma^{+}$and $\Gamma^{-}$, respectively. A rotation matrix $[\mathbf{R}(\mathbf{s})]$ is incorporated to the interpolation matrix to get the displacement discontinuity $\llbracket \mathbf{u}(\mathbf{s}) \rrbracket$ in the local coordinates system: 


$$
\llbracket \mathbf{u}(\mathbf{s}) \rrbracket=\left[\begin{array}{c}
\llbracket u_{n} \rrbracket \\
\llbracket u_{t} \rrbracket
\end{array}\right]=[\mathbf{R}(\mathbf{s})] .\left(\left[\mathbf{N}_{+}(\mathbf{s})\right]-\left[\mathbf{N}_{-}(\mathbf{s})\right]\right) \cdot\{\mathbf{U}\}=[\mathbf{D}(\mathbf{s})] .\{\mathbf{U}\}
$$

The discrete pressure fields are given by

$$
\begin{gathered}
p(\mathbf{x}) \approx[\mathbf{L}(\mathbf{x})] \cdot\{\mathbf{P}\} \\
p^{+}(\mathbf{s}) \approx\left[\mathbf{L}_{+}(\mathbf{s})\right] .\{\mathbf{P}\} \text { and } p^{-}(\mathbf{s}) \approx\left[\mathbf{L}_{-}(\mathbf{s})\right] .\{\mathbf{P}\}
\end{gathered}
$$

and

$$
p_{f}(\mathbf{s}) \approx\left[\mathbf{L}_{\mathbf{f}}(\mathbf{s})\right] .\left\{\mathbf{P}_{\mathbf{f}}\right\}
$$

where $\{\mathbf{P}\}$ and $\left\{\mathbf{P}_{\mathbf{f}}\right\}$ are the pressure nodal vectors in the porous medium and in the fracture pressure, respectively. $[\mathbf{L}(\mathbf{x})]$ and $\left[\mathbf{L}_{\mathbf{f}}(\mathbf{s})\right]$ are the matrices of the interpolation functions on $\Omega$ and $\Gamma$, respectively. $\left[\mathbf{L}_{+}(\mathbf{s})\right]$ and $\left[\mathbf{L}_{-}(\mathbf{s})\right]$ are the traces of $[\mathbf{L}(\mathbf{x})]$ on $\Gamma^{+}$and $\Gamma^{-}$, respectively.

To enforce the constraint of equality of the pressure across the interface (equation 2), we introduce the hydraulic Lagrange multipliers $q^{+}$and $q^{-}$. These new degrees of freedom are located on nodes 5 and 7 of the zero-thickness element. In order to satisfy the LBB (Ladyzenskaia-BabuškaBrezzi) condition [5], we choose hydraulic Lagrange multipliers that are constant per element:

$$
q^{ \pm}=[\mathbf{B}(\mathbf{s})] \cdot\left\{\mathbf{Q}^{ \pm}\right\}
$$

where $\left\{\mathbf{Q}^{+}\right\}$and $\left\{\mathbf{Q}^{-}\right\}$are the Lagrange multipliers nodal vectors and $[\mathbf{B}(\mathbf{s})]$ is the interpolation matrix constant per element.

\subsection{Variational equations and discrete formulation}

The variational form of equations (6), (10) and (8) is

$$
\begin{aligned}
\int_{\Omega}\left(\boldsymbol{\sigma}^{\prime}-b p \mathbf{I} \mathbf{d}\right): \boldsymbol{\varepsilon}(\hat{\mathbf{u}}) d \Omega+\int_{\Gamma}\left(\boldsymbol{\tau}^{\prime}-p_{f} \mathbf{n}\right) \llbracket \hat{\mathbf{u}} \rrbracket d \Gamma & = \\
\int_{\partial \Omega_{M}} \mathbf{f}_{\mathbf{M}} \cdot \hat{\mathbf{u}} d \Gamma & \forall \hat{\mathbf{u}} \in U_{a d} .
\end{aligned}
$$

where $U_{a d}$ is the set of the kinematically admissible displacement fields and $\mathbf{f}_{\mathbf{M}}$ the force boundary condition.

The variational forms of equations (7), (4) and (2), respectively, are 


$$
\begin{array}{cc}
\int_{\Omega} \frac{\partial}{\partial t}(\rho \Phi) \hat{p} d \Omega+\int_{\Omega} \rho_{0} \frac{\kappa}{\mu} \nabla p \cdot \nabla \hat{p} d \Omega= & \\
-\int_{\partial \Omega_{H}} f_{H} \hat{p} d \Gamma+\int_{\Gamma^{+}} q^{+} \hat{p} d \Gamma+\int_{\Gamma^{-}} q^{-} \hat{p} d \Gamma & \forall \hat{p} \in P_{a d} \\
\int_{\Gamma} \frac{\partial}{\partial t}\left(\rho \llbracket u_{n} \rrbracket\right) \hat{p}_{f} d \Gamma+\int_{\Gamma} \frac{\rho \llbracket u_{n} \rrbracket^{3}}{12 \mu} \frac{\partial p_{f}}{\partial s} \frac{\partial \hat{p}_{f}}{\partial s} d \Gamma & = \\
\int_{\partial \Gamma_{H}} f_{f, H} \hat{p}_{f} d s-\int_{\Gamma}\left(p^{+}+p^{-}\right) \hat{p}_{f} d \Gamma & \forall \hat{p}_{f} \in P_{f, a d} \\
\int_{\Gamma}\left(p^{+}-p_{f}\right) \hat{q}^{+} d \Gamma=\int_{\Gamma}\left(p^{-}-p_{f}\right) \hat{q}^{-} d \Gamma=0 \quad \forall \hat{q}^{+}, \hat{q}^{-}
\end{array}
$$

where $P_{a d}$ and $P_{f, a d}$ are the sets of the kinematically admissible pressure fields and $f_{H}$ and $f_{f, H}$ the flux boundary conditions, respectively on $\partial \Omega_{H} \subset$ $\partial \Omega$ and on $\partial \Gamma_{H} \subset \partial \Gamma$.

An explicit finite-difference scheme is used for the time discretization of (26) and (27). $t$ denotes the current time-step and $t^{-}$the last time-step $t-\Delta t$. At each time-step the following non-linear matrix system is solved using a fully-coupled Newton-Raphson method to compute $\{\mathbf{U}\},\{\mathbf{P}\},\left\{\mathbf{P}_{\mathbf{f}}\right\}$ and $\left\{\mathbf{Q}^{ \pm}\right\}$.

The discrete equation governing the mechanical behavior of the system is the following approximation of the variational form (25)

$$
\int_{\Omega}[\nabla \mathbf{N}]^{\mathbf{T}} \cdot \boldsymbol{\sigma}^{\prime} d \Omega-[\mathbf{C}] .\{\mathbf{P}\}+\int_{\Gamma}[\mathbf{D}]^{\mathbf{T}}\left(\boldsymbol{\tau}^{\prime}-\left\{\mathbf{P}_{\mathbf{f}}\right\} \mathbf{n}\right) d \Gamma=\left\{\mathbf{F}_{\mathbf{M}}^{\mathrm{ext}}\right\}
$$

Noting $[\mathbf{I d}]$ the identity matrix and $[\boldsymbol{\nabla N}]$ the matrix of the derivatives of the quadratic interpolation functions,

$$
[\mathbf{C}]=\int_{\Omega} b[\mathbf{\nabla N}]^{\mathbf{T}}[\mathbf{I d}][\mathbf{L}] d \Omega
$$

is the coupling matrix and $\left\{\mathbf{F}_{\mathbf{M}}^{\text {ext }}\right\}$ is the nodal vector of external forces.

The hydraulic behavior of the system is governed by the following set of equations

$$
\begin{array}{r}
{[\mathbf{C}]^{\mathbf{T}} \cdot\left(\{\mathbf{U}\}-\left\{\mathbf{U}_{t^{-}}\right\}\right)+[\mathbf{M}] \cdot\left(\{\mathbf{P}\}-\left\{\mathbf{P}_{t^{-}}\right\}\right)} \\
\Delta t\left([\mathbf{G}] .\{\mathbf{P}\}-\left\{\mathbf{F}_{\mathbf{H}}^{\text {ext }}\right\}+\left[\mathbf{E}^{+}\right] \cdot\left\{\mathbf{Q}^{+}\right\}+\left[\mathbf{E}^{-}\right] \cdot\left\{\mathbf{Q}^{-}\right\}\right)
\end{array}
$$




$$
\begin{array}{r}
\int_{\Gamma}\left[\mathbf{L}_{\mathbf{f}}\right]^{\mathbf{T}} \frac{1}{K_{f}}([\mathbf{D}] \cdot\{\mathbf{U}\})_{n}\left[\mathbf{L}_{\mathbf{f}}\right] \cdot\left(\left\{\mathbf{P}_{\mathbf{f}}\right\}-\{\mathbf{P}\}\right) d \Gamma+\int_{\Gamma}\left[\mathbf{L}_{\mathbf{f}}\right]^{\mathbf{T}}([\mathbf{D}] \cdot\{\mathbf{U}\})_{n} d \Gamma \\
=\Delta t\left(\int_{\Gamma}\left[\frac{\partial \mathbf{L}_{\mathbf{f}}}{\partial \mathbf{s}}\right]^{\mathbf{T}} \frac{([\mathbf{D}] \cdot\{\mathbf{U}\})_{n}^{3}}{12 \mu}\left[\frac{\partial \mathbf{L}_{\mathbf{f}}}{\partial \mathbf{s}}\right]^{\mathbf{T}} \cdot\left\{\mathbf{P}_{\mathbf{f}}\right\} d \Gamma\right) \\
-\Delta t\left(\left\{\mathbf{F}_{\mathbf{H f}}^{\mathbf{e x t}}\right\}-\left[\mathbf{E}_{\mathbf{f}}\right] \cdot\left(\left\{\mathbf{Q}^{+}\right\}+\left\{\mathbf{Q}^{-}\right\}\right)\right) \\
\left(\left[\mathbf{E}^{+}\right]^{\mathbf{T}} \cdot\{\mathbf{P}\}-\left[\mathbf{E}_{\mathbf{f}}\right]^{\mathbf{T}} \cdot\left\{\mathbf{P}_{\mathbf{f}}\right\}\right)+\left(\left[\mathbf{E}^{-}\right]^{\mathbf{T}} \cdot\{\mathbf{P}\}-\left[\mathbf{E}_{\mathbf{f}}\right]^{\mathbf{T}} \cdot\left\{\mathbf{P}_{\mathbf{f}}\right\}\right) \cdot=0
\end{array}
$$

which are the approximations of the variational forms (26), (27) and (28), respectively.

$[\mathbf{M}]$ and $[\mathbf{G}]$ are the compressibility and the permeability matrix of the porous medium discontinuity, respectively,

$$
\begin{gathered}
{[\mathbf{M}]=\int_{\Omega}[\mathbf{L}]^{\mathbf{T}}\left(\frac{\Phi_{0}}{K_{f}}-\frac{1}{M}\right)[\mathbf{L}] d \Omega} \\
{[\mathbf{G}]=\int_{\Omega}[\nabla \mathbf{L}]^{\mathbf{T}} \frac{\kappa}{\mu}[\nabla \mathbf{L}] d \Omega .}
\end{gathered}
$$

$\left\{\mathbf{F}_{\mathbf{H}}^{\text {ext }}\right\}$ and $\left\{\mathbf{F}_{\mathbf{H f}}^{\text {ext }}\right\}$ are the nodal vectors of external flow.

$\left[\mathbf{E}^{+}\right],\left[\mathbf{E}^{-}\right]$and $\left[\mathbf{E}_{\mathbf{f}}\right]$ are the exchange matrices

$$
\left[\mathbf{E}^{ \pm}\right]=\int_{\Gamma}\left[\mathbf{L}^{ \pm}\right]^{\mathbf{T}} \cdot[\mathbf{B}] d \Gamma
$$

and

$$
\left[\mathbf{E}_{\mathbf{f}}\right]=\int_{\Gamma}\left[\mathbf{L}_{\mathbf{f}}\right]^{\mathbf{T}} \cdot[\mathbf{B}] d \Gamma .
$$

\section{Results and benchmarks}

\subsection{Problem definition}

In this section, we apply our model to the KGD fracture problem (figure 5 ). We consider the injection of an incompressible fluid in a fracture in plane strain conditions in an infinite permeable medium. The injection rate per unit of length $Q_{0}$ in the well is assumed to be constant. The symmetry of the problem allows us to model only half of the space. The $x$-axis coincides with the fracture path; the origin of the $x$-axis is at the injection point. 


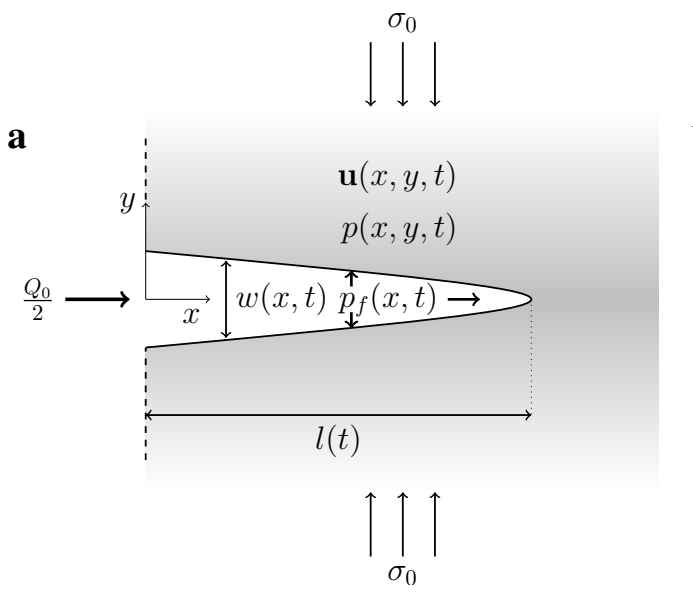

b

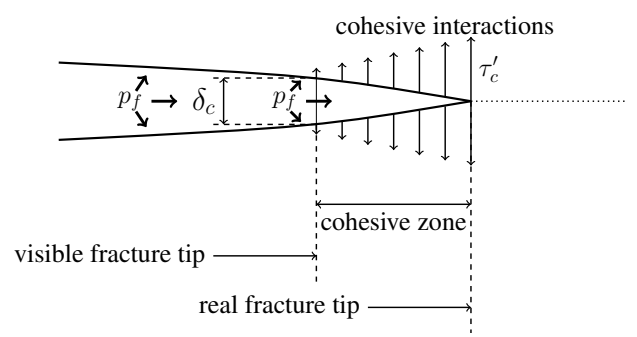

Figure 5: KGD fracture problem (a) and representation of the cohesive zone (b).

\begin{tabular}{ccc} 
Young's modulus & $E$ & $17 \mathrm{GPa}$ \\
Poisson ratio & $\nu$ & 0.2 \\
\hline Fracturing energy & $G_{c}$ & $120 \mathrm{~Pa} . \mathrm{m}$ \\
Critical stress & $\tau_{c}$ & $1.25 \mathrm{MPa}$ \\
\hline Biot coefficient & $b$ & 0.75 \\
Biot Modulus & $M$ & $68.7 \mathrm{MPa}$ \\
Porosity & $\Phi$ & 0.2 \\
\hline Injection rate & $Q_{0}$ & $0.001 \mathrm{~m}^{3} . \mathrm{s}^{-1}$ \\
Far-field stress & $\sigma_{0}$ & $3.7 \mathrm{MPa}$
\end{tabular}

Table 1: Input parameters

A compressive far-field stress $\sigma_{0}$ acts perpendicularly to the fracture. $l(t)$ denotes the fracture length.

The material parameters used for all the numerical simulations are given in table 1. The influence of the hydraulic properties is investigated. As the mesh density is essentially governed by the cohesive zone parameters to ensure numerical convergence, the same mesh (figure 6) was used in all the simulations. The initial configuration was obtained by breaking the very first interface element near the injection point. The length of this element is 5 $\mathrm{cm}$. Its aperture and its cohesive energy were set to zero in order to allow the free opening and the fluid injection.

In all simulations, we monitor the flow rate leaving the fracture. We check whether this flow rate is proportional to $t^{-1 / 2}$ and back-calculate $C_{L}$ by linear regression. Table 2 gives the leak-off coefficients used for comparison with the simulations.

All the simulations presented in this section are run with the finite element 


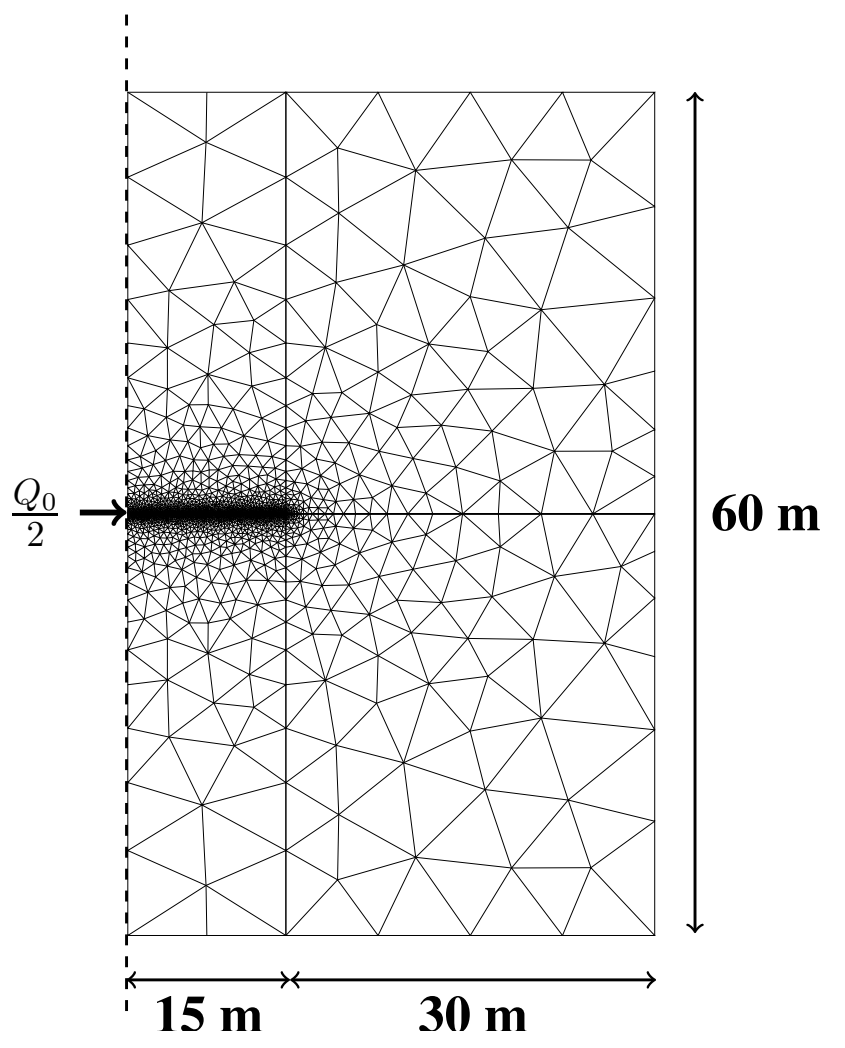

Figure 6: Mesh used in the simulations.

software Code_Aster [18].

\subsection{Storage-toughness dominated regime}

To investigate the influence of intrinsic permeability of the surrounding porous medium, we use a viscosity $\mu=0.0001 \mathrm{~Pa} . \mathrm{s}$, the cohesive parameters given in table 2 and a far-field stress $\sigma_{0}=3.7 \mathrm{MPa}$. With these parameters the fracture propagation evolves in the toughness-dominated regime (dimensionless viscosity $\mathcal{M}=\mathcal{K}^{-4}=1.42 \times 10^{-2}$ ).

We perform numerical simulations for two permeabilities $\kappa=1.10^{-16} \mathrm{~m}^{2}$ and $\kappa=1.10^{-15} \mathrm{~m}^{2}$. For these permeabilities, the characteristic times of leak-off are $\sim 10^{7} \mathrm{~s}$ and $\sim 10^{3} \mathrm{~s}$, respectively. We simulate injections of 14 $\mathrm{s}$ and $20 \mathrm{~s}$, respectively, thus ensuring that the fracture propagation stays in the storage regime. The results obtained by simulation are displayed in figures 7 and 8 together with the analytical solution for the toughness-storage regime $[7,25]$ (near- $K$ solution). The agreement between the numerical and the analytical solutions is very good. Figure 7 also shows the extent of the fracture process zone. The cohesive zone length is constant during the simulations and is approximately $0.8 \mathrm{~m}$ long. 


\begin{tabular}{cccccc}
$\kappa\left(\mathrm{m}^{2}\right)$ & $\mu($ Pa.s $)$ & $\sigma_{0}(\mathrm{MPa})$ & $C_{L}\left(\mathrm{~m} . \mathrm{s}^{-1 / 2}\right)$ & Regime & Figures \\
\hline $1.10^{-16}$ & $1.10^{-4}$ & 3.7 & $1.47 \times 10^{-5}$ & near- $K$ & $7-8-10-9$ \\
$1.10^{-15}$ & $1.10^{-4}$ & 3.7 & $6.28 \times 10^{-5}$ & near- $K$ & $7-8-10-9$ \\
$5.10^{-15}$ & $1.10^{-4}$ & 5.0 & $2.0 \times 10^{-4}$ & near- $\widetilde{K}$ & $11-12-13$ \\
\hline $1.10^{-12}$ & 0.1 & 3.7 & 0 & $M$ & $17-18-19-20$ \\
$5.10^{-12}$ & 0.1 & 7.2 & $1.6 \times 10^{-4}$ & $\widetilde{M}$ & $21-22-23$
\end{tabular}

Table 2: Leak-off coefficients corresponding to the permeabilities and viscosities used in the simulations and asymptotic propagation regimes reached with these leak-off coefficients. The fifth column reports the figures in which we display the associated asymptotic solutions.
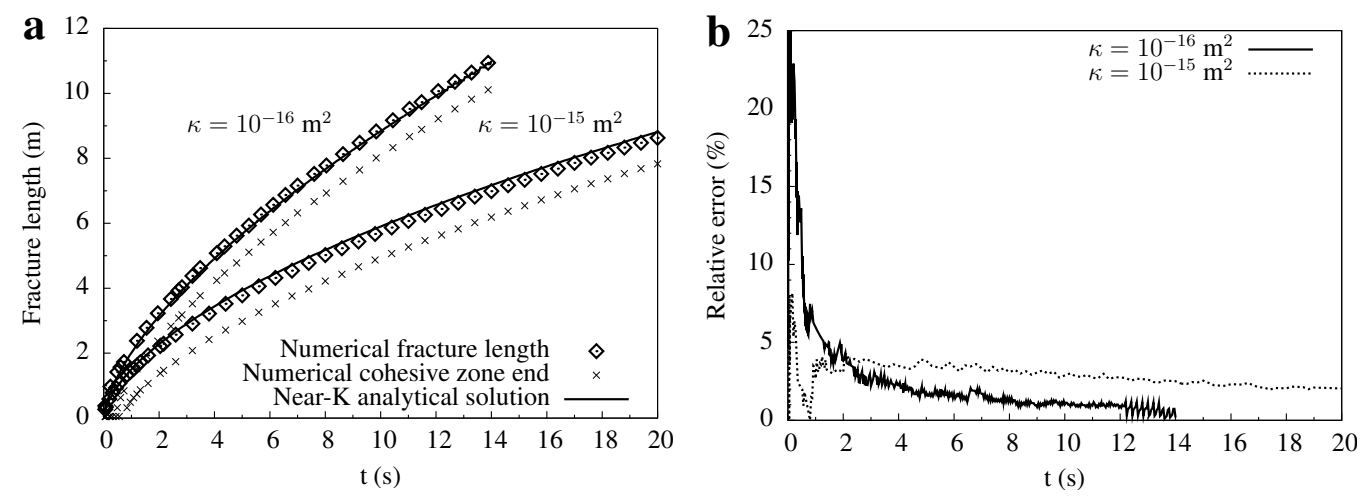

Figure 7: Fracture length (a) and relative error (b) for $\mu=1.10^{-4}$ Pa.s. The analytical solutions are the asymptotic solutions in the storage-toughness dominated regime [7].
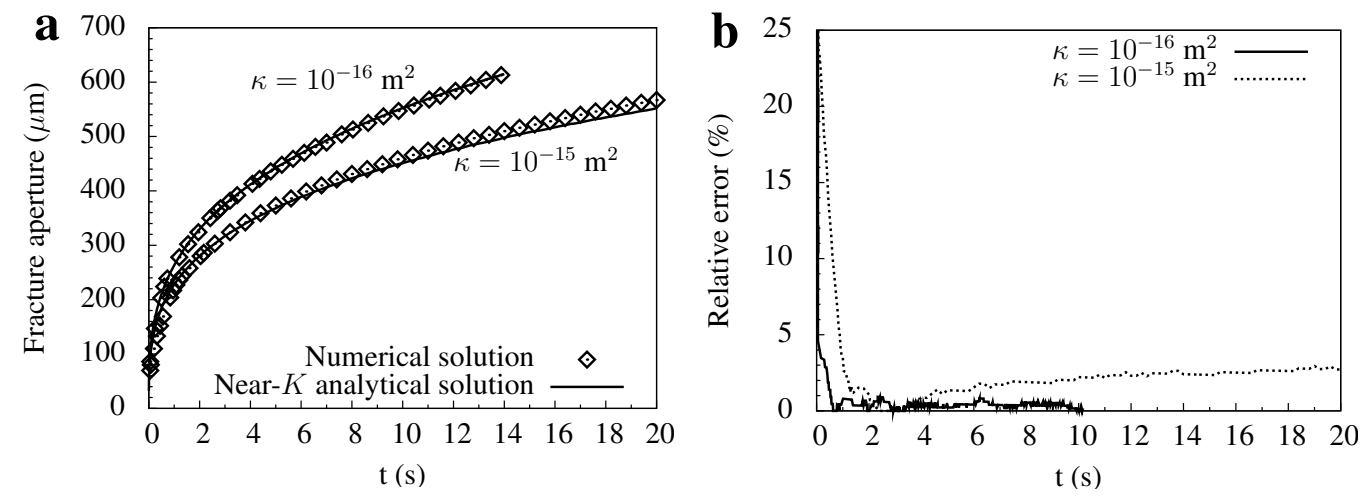

Figure 8: Fracture aperture (a) and relative error (b) for $\mu=1.10^{-4}$ Pa.s. The analytical solutions are the asymptotic solutions in the storage-toughness dominated regime [7].

Figure 9 shows the fracture profile for the two considered permeabilities at $t=10 \mathrm{~s}$. Far from the tip we have a perfect match between numerical and analytical computations. Because the two models use different propagation 
criteria, the profiles have different shapes at the fracture tip. Figure 9 also displays the normal effective stress across the fracture. It shows the extent of the process zone, where the cohesive forces are positive. As expected, the normal effective stress is equal to zero in the broken part of the fracture and is equal to the far-field stress ahead of the fracture tip.
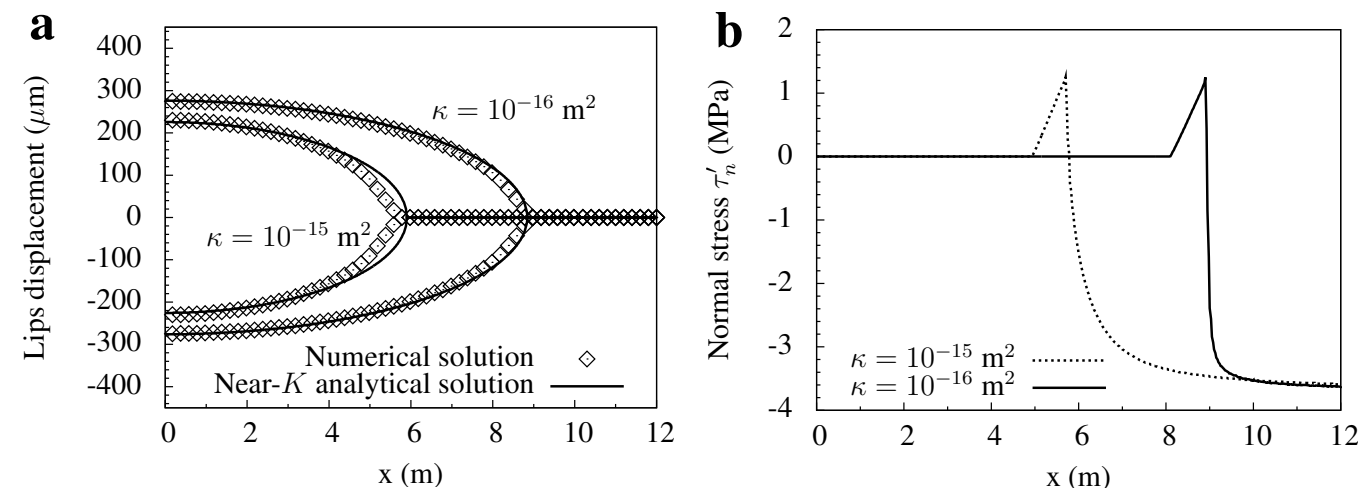

Figure 9: Fracture profile (a) and normal effective stress across the fracture (b) for $\mu=$ $1.10^{-4}$ Pa.s at $t=10 \mathrm{~s}$. The analytical solutions are the asymptotic solutions in the storage-toughness dominated regime [7].

Figure 10 shows the net fluid injection pressure $\left(p_{f}-\sigma_{0}\right)$ versus time. The pressure obtained from numerical simulations is always greater than the pressure obtained analytically. While the analytical model neglects the hydro-mechanical coupled behavior of the surrounding porous medium, our model takes this coupling into account. The fluid leak-off induces an increase of pore pressure in the porous medium and therefore stiffens the surrounding medium and generates an additional compressive stress: the so-called back-stress [45, 28]. Indeed, if we remove the hydro-mechanical coupling by artificially imposing a Biot coefficient equal to zero in the mechanical equilibrium equation of the medium, the analytical and numerical solutions are in very good agreement (circles in figure 10). As Detournay et al. [15], we observe that poroelastic effects induce a significant increase of the injection pressure but have no effect on the length and the aperture of the fracture.

\subsection{Leak-off-toughness dominated regime}

Keeping the other parameters fixed, we now perform a simulation for a higher permeability $\kappa=5.10^{-15} \mathrm{~m}^{2}$ and a higher confining stress $\sigma_{0}=5$ $\mathrm{MPa}$ in order to increase the leak-off coefficient. For this permeability the characteristic time of leak-off is $\sim 1 \mathrm{~s}$. The total simulation time is $100 \mathrm{~s}$. Therefore the fracture reaches very quickly the leak-off (near- $\widetilde{K}$ ) propagation regime. Figures 11 and 12 show the numerical fracture length and aperture. 

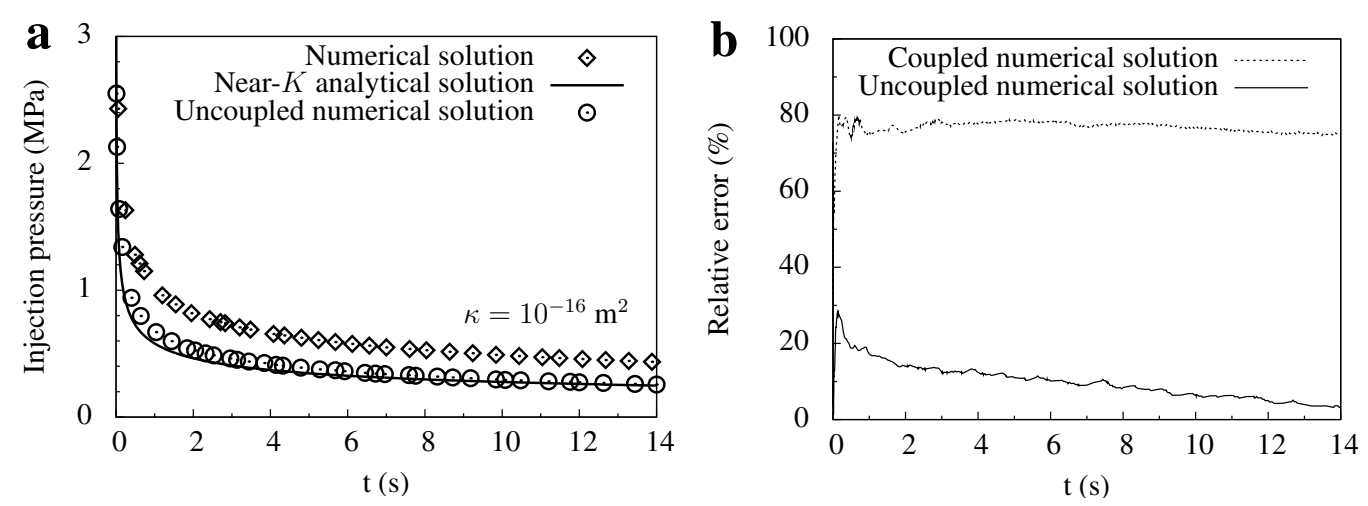

Figure 10: Injection net pressure (a) and relative error (b) for $\mu=1.10^{-4}$ Pa.s. The analytical solution is the asymptotic solution in the storage-toughness dominated regime [7]. The uncoupled numerical solution is the result of a simulation in which the hydromechanical coupling in the porous medium is artificially removed.

They display the results of two computations: with a bi-dimensional fluid diffusion and with an unidimensional fluid diffusion in the porous medium.

In the case of a $2 \mathrm{D}$ diffusion, the permeability of the surrounding medium is isotropic $\left(\kappa_{x}=\kappa_{y}=5.10^{-15} \mathrm{~m}^{2}\right)$ while in order to have a $1 \mathrm{D}$ diffusion, we choose a strongly anisotropic permeability $\left(\kappa_{y}=5.10^{-15} \mathrm{~m}^{2}\right.$ and $\kappa_{y}=$ $1.10^{-16} \mathrm{~m}^{2} \ll \kappa_{y}$ ). Monitoring the computed leak-off at a given point in the fracture shows that the assumption of unidimensional diffusion is no longer relevant for $\kappa=5.10^{-15} \mathrm{~m}^{2}$ if the permeability is kept anisotropic. Figure 14 shows the fluid pressure distribution at $t=100 \mathrm{~s}$. For an isotropic permeability, the diffusion pattern is clearly bi-dimensional and the fluid penetration front goes further than the crack tip. However,the diffusion pattern shows that the unidimensional diffusion hypothesis is valid in the case of the anisotropic permeability. Moreover, the leak-off at a given point in the fracture is proportional to $t^{-1 / 2}$ and the Carter's law proves to be a good approximation.

Figures 11 and 12 show the near- $\widetilde{K}$ fracture length and aperture [7, 25]. The storage-toughness (near- $K$ ) solution [7] is also plotted but, as expected, diverges very quickly from the numerical solution. Figure 13 displays the relative error between the near- $\widetilde{K}$ analytical solution and the numerical solution in the case of an unidimensional diffusion. The agreement between the leakoff-toughness solution and the numerical one is very good when we impose an unidimensional leak-off. However, as in the storage-toughness regime, poroelastic effects induce a significant increase of the injection pressure. 


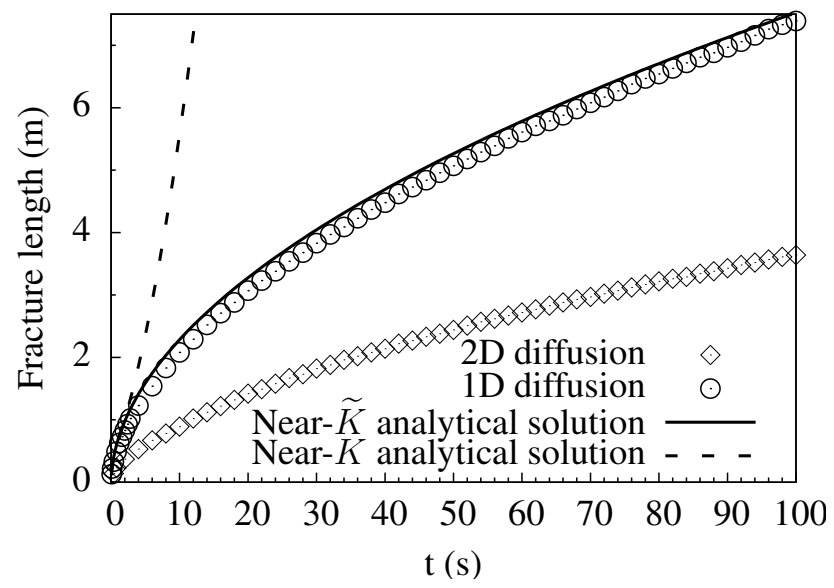

Figure 11: Fracture length for $\mu=1.10^{-4}$ Pa.s, $\kappa=5.10^{-15} \mathrm{~m}^{2}$ and $\sigma_{0}=5 \mathrm{MPa}$. The analytical solutions are the asymptotic solution in the storage-toughness dominated regime (dashed line) and the leak-off-toughness dominated regime (solid line) [7].

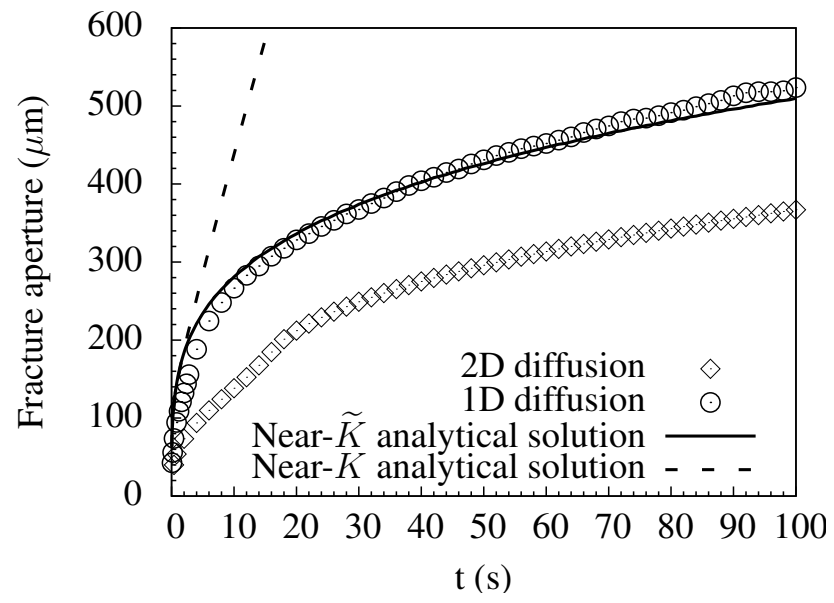

Figure 12: Fracture aperture for $\mu=1.10^{-4}$ Pa.s, $\kappa=5.10^{-15} \mathrm{~m}^{2}$ and $\sigma_{0}=5 \mathrm{MPa}$. The analytical solutions are the asymptotic solution in the storage-toughness dominated regime (dashed line) and the leak-off-toughness dominated regime (solid line) [7].

\subsection{Storage-viscosity dominated regime}

Figures 15 and 16 show the effect of the fluid viscosity on the fracture. We use the same hydraulic conductivity $k=\kappa \mu^{-1} \rho g=1.10^{-7} \mathrm{~m} \cdot \mathrm{s}^{-1}$ in all numerical simulations. The far-field stress is $3.7 \mathrm{MPa}$. For a low viscosity, the pressure is uniform in the fracture (figure 16). As we increase the viscosity, the pressure profile is no longer uniform. For higher viscosities, the pressure is much higher at the fracture mouth but lower at the fracture tip, therefore leading to a fracture which is wider near the injection point (figures 15 and 18) but shorter (figure 17). 


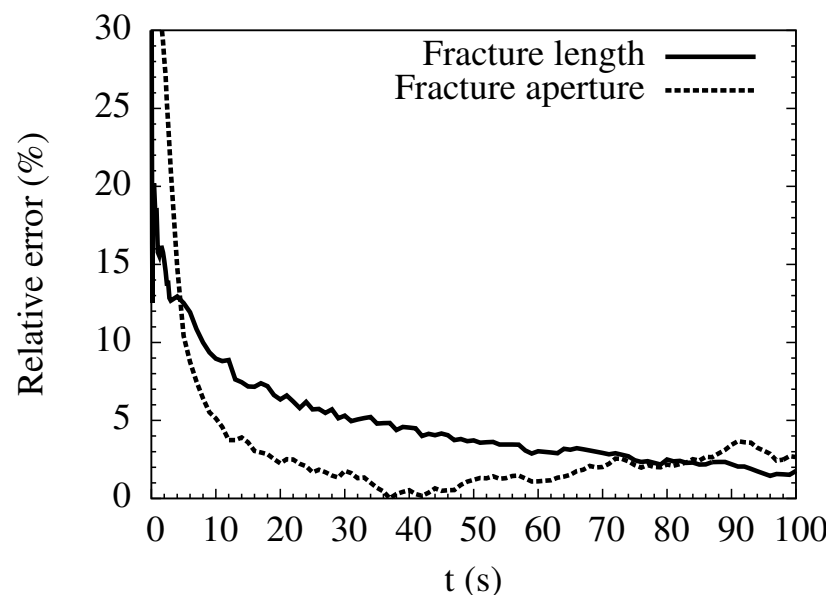

Figure 13: Relative error of the fracture length and the fracture aperture for $\mu=1.10^{-4}$ Pa.s, $\kappa=5.10^{-15} \mathrm{~m}^{2}$ and $\sigma_{0}=5 \mathrm{MPa}$. The numerical solution is the result of a $1 \mathrm{D}$ diffusion computation $\left(\kappa_{y}=5 \cdot 10^{-15} \mathrm{~m}^{2}\right.$ and $\left.\kappa_{x}=1.10^{-16} \mathrm{~m}^{2}\right)$. The reference solution is the asymptotic solution in the leak-off-toughness dominated regime (solid line) [7].

a - 2D diffusion

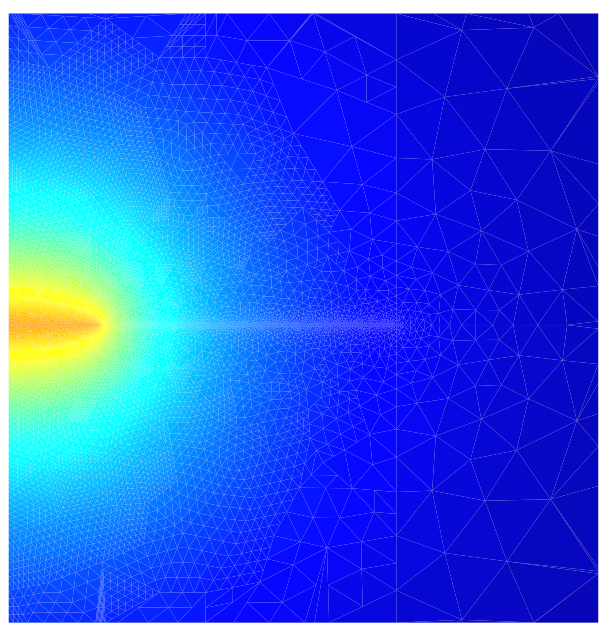

$-1.84 e+05 \quad 4.69 e+06$ b - 1D diffusion

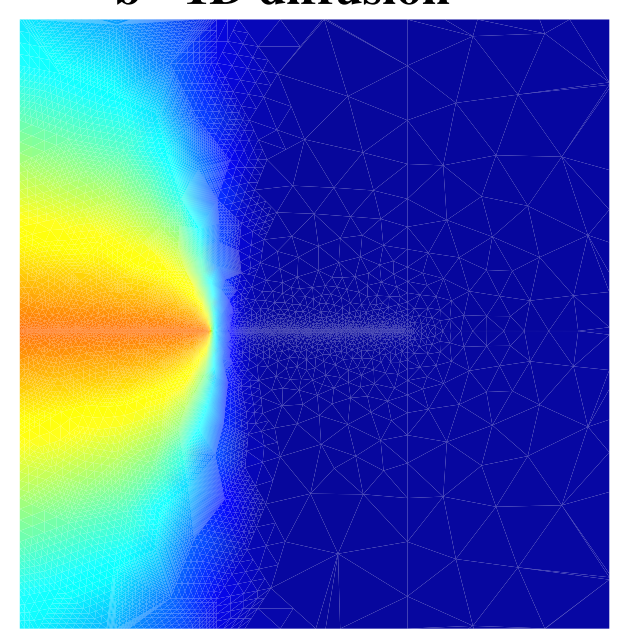

$9.57 e+06$

Figure 14: Fluid pressure distributions (in $\mathrm{Pa}$ ) at $t=100 \mathrm{~s}$ in the case of $2 \mathrm{D}$ diffusion (a) and $1 \mathrm{D}$ diffusion (b).

In figures 17, 18, 19 and 20 we compare the numerical results for $\mu=0.1$ Pa.s and $\kappa=1.10^{-15} \mathrm{~m}^{2}$ with the corresponding analytical solution in the storage-toughness regime [3]. In that case the dimensionless viscosity is $\mathcal{M}=$ 14.2 and the dimensionless toughness is $\mathcal{K}=0.51$. According to Adachi et al. [3], $\mathcal{K} \lesssim 1$ is a sufficient validity condition to be in the storage-toughness 


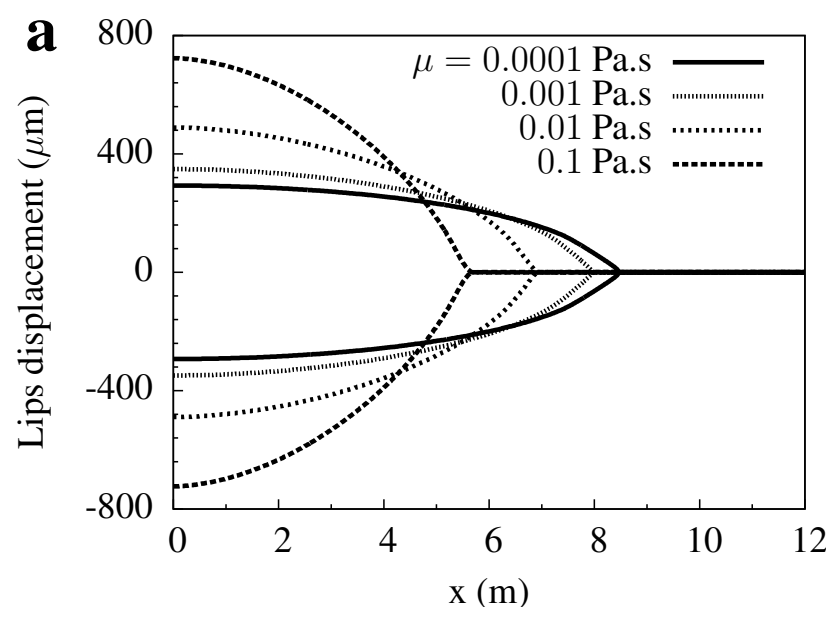

Figure 15: Fracture opening profiles at $t=20 \mathrm{~s}$ for the same hydraulic conductivity $k=1.10^{-7} \mathrm{~m} \cdot \mathrm{s}^{-1}$ of the porous medium but for different fluid viscosities.

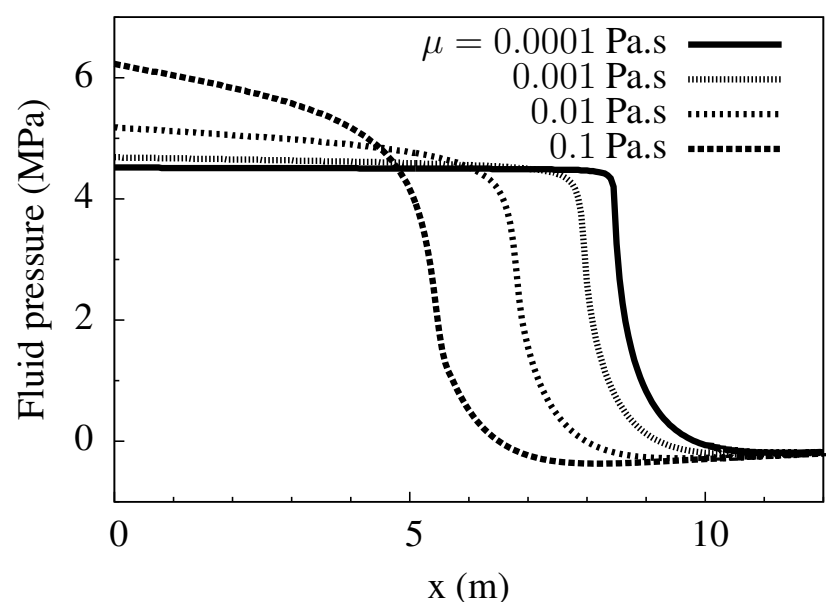

Figure 16: Fluid pressure profile at $t=20 \mathrm{~s}$ for the same hydraulic conductivity $k=1.10^{-7}$ $\mathrm{m} . \mathrm{s}^{-1}$ of the porous medium but for different fluid viscosities.

regime. We display the results of a fully coupled numerical simulation and of an uncoupled computation. For the uncoupled simulation, the hydromechanical coupling was artificially removed in the mechanical equilibrium equation. The agreement between the numerical uncoupled simulation and the asymptotic solution is very good (figure 20) . However, the agreement is not as good in the fully coupled case. The back-stress induces an increase of the fluid pressure at the fracture mouth (figure 19) and a decrease of the fracture aperture (figure 18). 


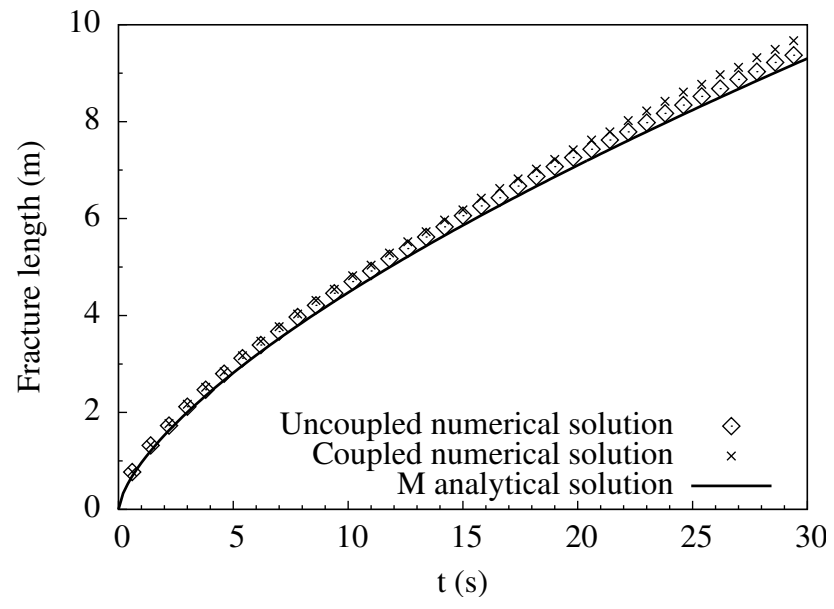

Figure 17: Fracture length for $\mu=0.1$ Pa.s and $\kappa=1.10^{-15} \mathrm{~m}^{2}$. Both the results of a fully coupled simulation and an uncoupled simulation are displayed. The analytical solution (solid line) is the storage-viscosity asymptotic solution [3].

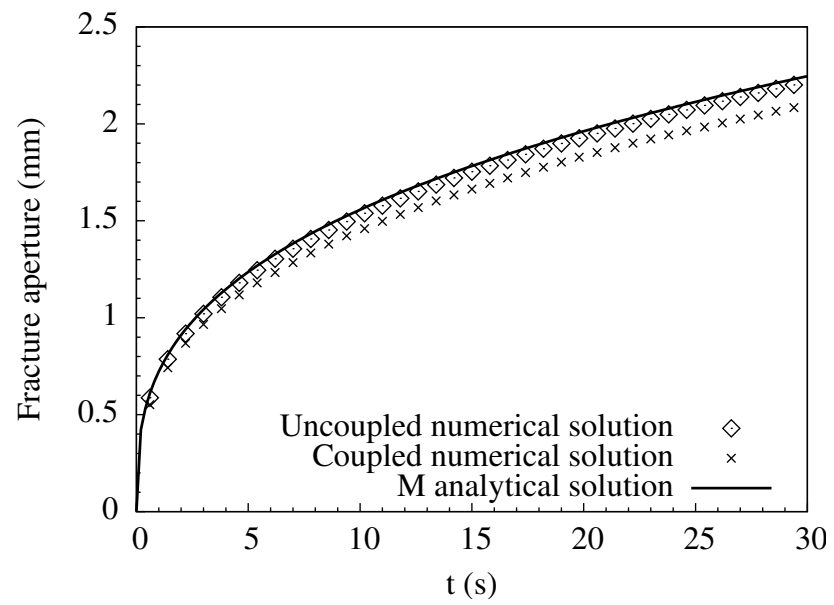

Figure 18: Fracture aperture for $\mu=0.1$ Pa.s and $\kappa=1.10^{-15} \mathrm{~m}^{2}$. Both the results of a fully coupled simulation and an uncoupled simulation are displayed. The analytical solution (solid line) is the storage-viscosity asymptotic solution [3].

\subsection{Leak-off-viscosity dominated regime}

We now perform simulations with the following parameters: $\kappa=5.10^{-12}$ $\mathrm{m}^{2}, \mu=0.1$ Pa.s and $\sigma_{0}=7.2 \mathrm{MPa}$. The total simulation time is $200 \mathrm{~s}$. Figures 21 and 22 and 23 show the fracture length, mouth aperture and net injection pressure in three different cases. The first case is the full computation of fracture propagation in a poroelastic medium. In the second one, the permeability of the surrounding medium is strongly anisotropic $\left(\kappa_{y}=\kappa=5.10^{-12}\right.$ $\mathrm{m}^{2}$ and $\left.\kappa_{x}=1.10^{-16}\right)$ in order to have an unidimensional diffusion and the 


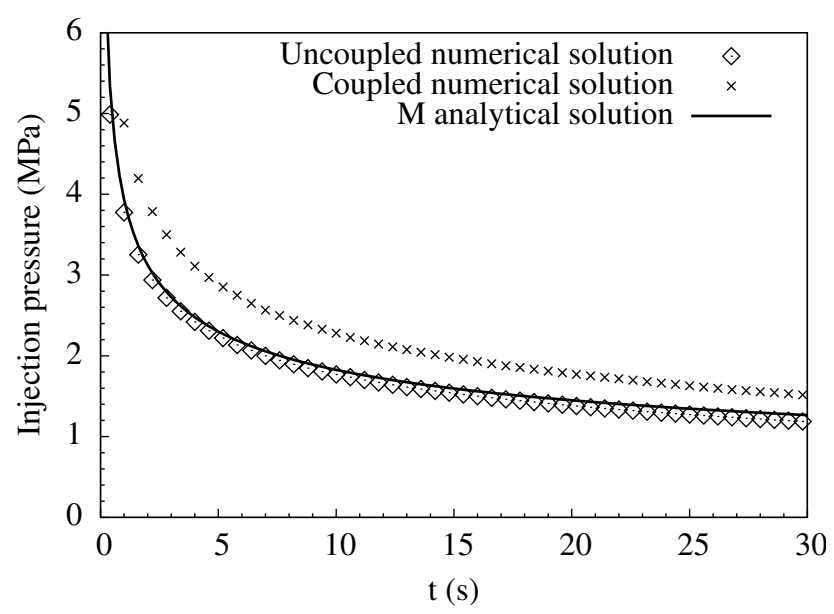

Figure 19: Injection pressure for $\mu=0.1$ Pa.s and $\kappa=1.10^{-15} \mathrm{~m}^{2}$. Both the results of a fully coupled simulation and an uncoupled simulation are displayed. The analytical solution (solid line) is the storage-viscosity asymptotic solution [3].

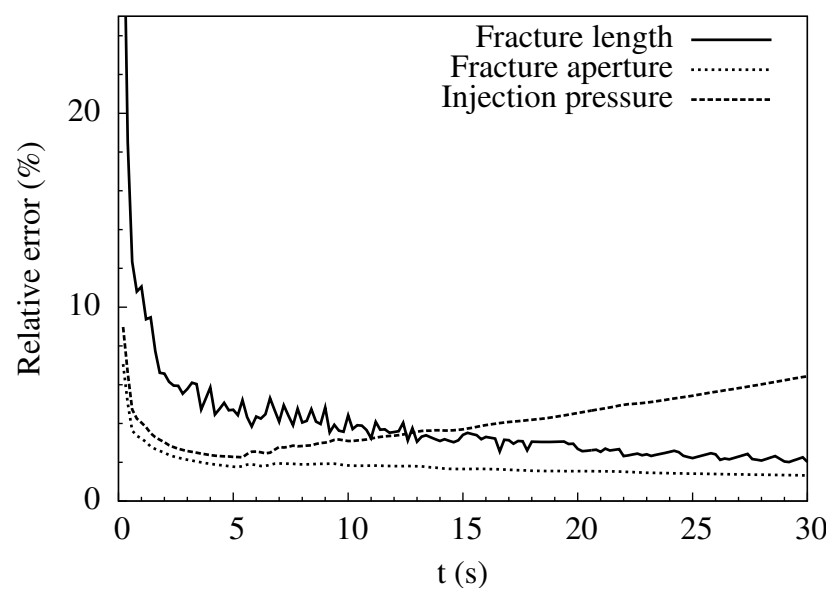

Figure 20: Relative error of the fracture length, the fracture aperture and the injection pressure for $\mu=0.1$ Pa.s and $\kappa=1.10^{-15} \mathrm{~m}^{2}$. The numerical solution is the result of an uncoupled computation and the reference solution is the storage-viscosity asymptotic solution [3].

porous medium is poroelastic. In the third case, the permeability is strongly anisotropic $\left(\kappa_{y}=\kappa=5.10^{-12} \mathrm{~m}^{2}\right.$ and $\left.\kappa_{x}=1.10^{-16} \mathrm{~m}^{2}\right)$ and the hydromechanical coupling is removed in the mechanical equilibrium equation. The comparison of these three computations shows that both the diffusion pattern and the back-stress have a strong effect on fracture propagation.

We compare the solution in the unidimensional uncoupled case with the $\widetilde{M}$ analytical solution [3] in figures 21, 22 and 23 and observe a good agreement between the numerical and the analytical solutions. The characteristic 
time of leak-off is $\sim 50 \mathrm{~s}$.

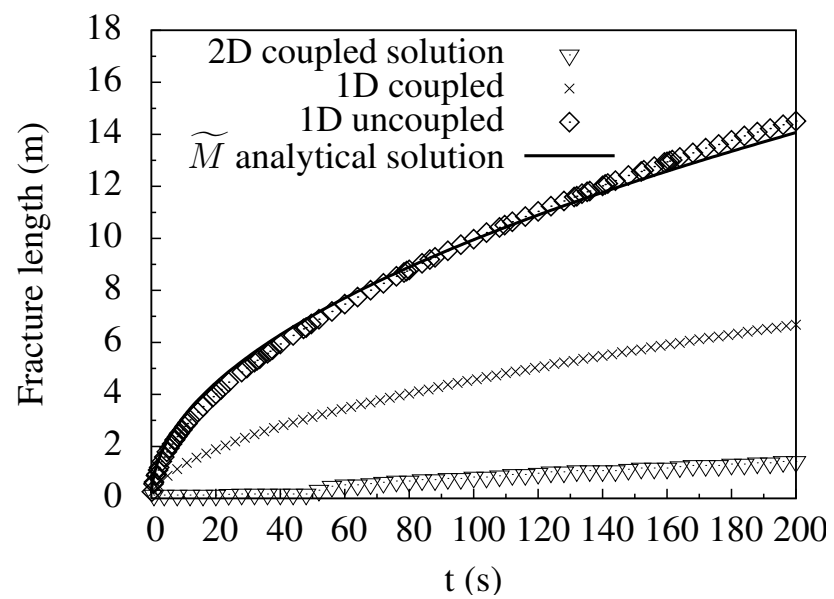

Figure 21: Fracture length for $\kappa=5.10^{-12} \mathrm{~m}^{2}, \mu=0.1 \mathrm{~Pa} . \mathrm{s}$ and $\sigma_{0}=7.2 \mathrm{MPa}$. The analytical solution (solid line) is the leak-off-viscosity asymptotic solution [3].

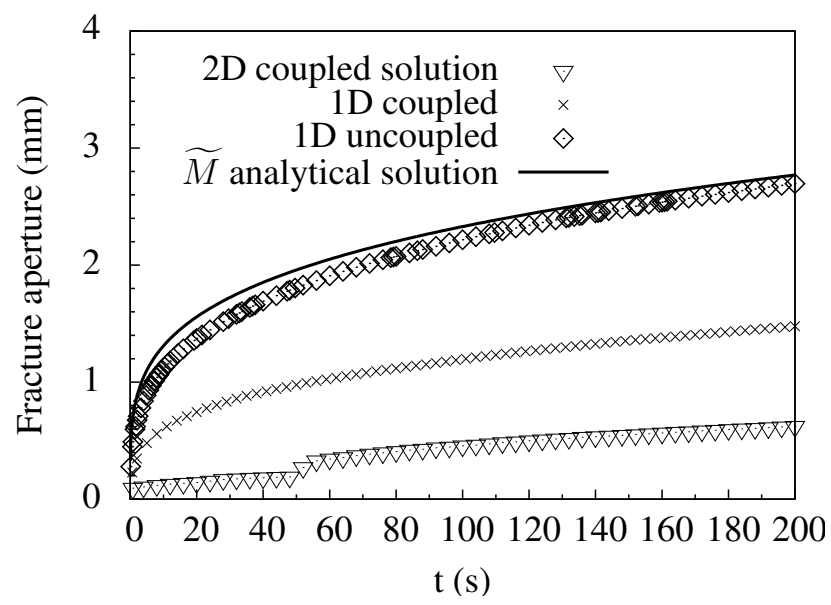

Figure 22: Fracture aperture for $\kappa=5.10^{-12} \mathrm{~m}^{2}, \mu=0.1$ Pa.s and $\sigma_{0}=7.2 \mathrm{MPa}$. The analytical solution (solid line) is the leak-off-viscosity asymptotic solution [3]. 


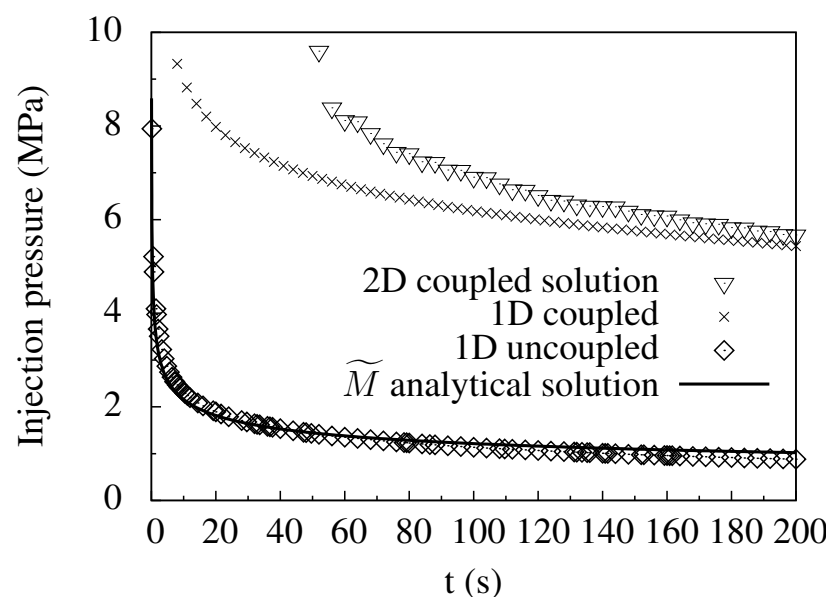

Figure 23: Injection net pressure for $\kappa=5.10^{-12} \mathrm{~m}^{2}, \mu=0.1$ Pa.s and $\sigma_{0}=7.2 \mathrm{MPa}$. The analytical solution (solid line) is the leak-off-viscosity asymptotic solution [3].

\section{Conclusions}

In this paper we presented a finite element-based numerical framework to model fluid-driven fracture propagation in a poroelastic medium. We developed a zero thickness interface element to mesh the fracture and its path. This element can easily be implemented in conjunction with bulk hydromechanical finite elements. The main coupled phenomena are taken into account: fluid flow in the fracture, 2D fluid diffusion in the porous medium, hydro-mechanical coupled behavior of the porous medium and fracture propagation. The pressure and the displacement fields can be computed in the fracture and in the whole porous medium.

The effect of the porous medium permeability and of the fluid viscosity were investigated. The numerical results were confronted to asymptotic analytical solutions for hydraulic fracture propagation. The four corners of the dissipation-storage plan were explored. The developed framework captured very well the asymptotic solution in all the propagation regimes under the assumptions of unidimensional leak-off and insensitivity of the mechanical response of the porous medium to the fluid pressure.

In the toughness dominated regime, when the rock stiffening due to fluid diffusion was taken into account into the numerical model the computed injection pressure was higher than the analytical one. However, this poroelastic effect has no effect on the fracture length and aperture. In the storageviscosity dominated regime, the pressure profile in the fracture is no longer uniform. Therefore the effect of the back-stress is higher at the mouth of the fracture: the fracture is longer but the fracture aperture is smaller.

In the analytical model, leak-off is governed by Carter's law while the 2D 
diffusion equation is fully solved in the surrounding medium by the numerical model. Carter's law can be derived from the diffusion equation assuming the diffusion is unidimensional. For low permeabilities, this hypothesis is confirmed and Carter's law was retrieved numerically. Nevertheless, for higher permeabilities, the fluid diffusion is no longer unidimensional and the fracture propagation is slower.

The zero-thickness element method gives access to a finer description of the fluid diffusion in the porous medium and of its coupled mechanical response. Moreover the cohesive zone model proved to be a convenient way to avoid both stress and pressure singularities at the fracture tip. The mesh must be dense enough at the fracture tip to guarantee the numerical convergence of the cohesive zone model and to describe finely the pressure profile, especially when it is not uniform. Although we showed results for a straight fracture in plane-strain conditions only, the method could be extended to 3D and to more complex geometries. Another way to deal with this problem would be to include the tip singularities particular to the hydraulic fracture problem directly in the extended finite element method (X-FEM) framework.

\section{Acknowledgements}

The authors wish to thank Dr. Roméo Fernandes from EDF R\&D for his invaluable help in the implementation of the numerical simulations. The comments from Dr. Patrick Massin (Lamsid) and Dr. Matthieu Vandamme (Navier) have also been greatly appreciated.

[1] H Abe, T Mura, and LM Keer. Growth-rate of a penny-shaped crack in hydraulic fracturing of rocks. J. Geophys. Res., 81(29):5335-5340, 1976.

[2] A. Adachi, E. Siebrits, A. Peirce, and J. Desroches. Computer simulation of hydraulic fractures. Int. J. Rock Mech. Min. Sci., 44(5):739-757, JUL 2007.

[3] JI Adachi and E Detournay. Plane strain propagation of a hydraulic fracture in a permeable rock. Eng. Fract. Mech., 75(16):4666-4694, nov 2008 .

[4] GI Barenblatt. The mathematical theory of equilibrium cracks in brittle fracture. Adv. Appl. Mech., 7(55-129):55-130, 1962.

[5] KJ Bathe and F Brezzi. Stability of finite element mixed interpolation for contact problems. Rend. Mat. Acc. Lincei, 12(9):167-183, 2001. 
[6] TJ Boone and AR Ingraffea. A numerical procedure for simulation of hydraulically-driven fracture propagation in poroelastic media. Int. J. Numer. Anal. Methods Geomech., 14(1):27-47, 1990.

[7] AP Bunger, E Detournay, and DI Garagash. Toughness-dominated hydraulic fracture with leak-off. Int. J. Fract., 134(2):175-190, jul 2005.

[8] ED Carter. Optimum fluid characteristics for fracture extension. Drilling and Production Practices, pages 261-270, 1957.

[9] M Charlotte, J Laverne, and JJ Marigo. Initiation of cracks with cohesive force models: a variational approach. Eur. J. Mech. A/Solids, 25(4):649-669, 2006.

[10] Z Chen, AP Bunger, X Zhang, and RG Jeffrey. Cohesive zone finite element-based modeling of hydraulic fractures. Acta Mech. Solida Sinica, 22(5, Sp. Iss. SI):443-452, oct 2009.

[11] Alfred Cornec, Ingo Scheider, and Karl-Heinz Schwalbe. On the practical application of the cohesive model. Engineering Fracture Mechanics, 70(14):1963 - 1987, 2003. jce:title¿Cohesive Modelsi/ce:title ¿.

[12] O Coussy. Poromechanics. John Wiley \& Sons Inc, 2004.

[13] J Desroches, E Detournay, B Lenoach, P Papanastasiou, JRA Pearson, $\mathrm{M}$ Thiercelin, and A Cheng. The crack tip region in hydraulic fracturing. Proc. Roy. Soc. London Series A, 447(1929):39-48, oct 81994.

[14] E Detournay. Propagation regimes of fluid-driven fractures in impermeable rocks. Int. J. Geomech., 4:35-45, 2004.

[15] E Detournay, AHD Cheng, and JD McLennan. A poroelastic PKN hydraulic fracture model based on an explicit moving mesh algorithm. ASME J. Ener. Res. Tech., 112(4):224-230, DEC 1990.

[16] DS Dugdale. Yielding of steel sheets containing slits. J. Mech. Phys., 8(2):100-104, 1960.

[17] M. Economides and K. Nolte. Reservoir Stimulation. Wiley, 2000.

[18] EDF R\&D. Code_Aster. www. code-aster.org.

[19] M Elices, GV Guinea, J Gomez, and J Planas. The cohesive zone model: advantages, limitations and challenges. Eng. Fract. Mech., 69(2):137$163,2002$. 
[20] A Ern and S Meunier. A posteriori error analysis of Euler-Galerkin approximations to coupled elliptic-parabolic problems. ESAIM: M2AN, 43(2):353-375, 2009.

[21] DI Garagash. Plane-strain propagation of a fluid-driven fracture during injection and shut-in: Asymptotics of large toughness. Eng. Fract. Mech., 74(16):456-481, nov 2007.

[22] J Geertsma and F De Klerk. A rapid method of predicting width and extent of hydraulically induced fractures. J. Pet. Technol., 21(12):15711581, 1969.

[23] C Guiducci, A Pellegrino, JP Radu, F Collin, and R Charlier. Numerical modeling of hydro-mechanical fracture behavior. In Numerical Models in Geomechanics-NUMOG VIII, pages 293-299, 2002.

[24] A. Hillerborg, M. Modéer, and P.E. Petersson. Analysis of crack formation and crack growth in concrete by means of fracture mechanics and finite elements. Cement Concrete Res., 6(6):773-781, 1976.

[25] J Hu and DI Garagash. Plane-Strain Propagation of a Fluid-Driven Crack in a Permeable Rock with Fracture Toughness. J. Eng. Mech.ASCE, 136(9):1152-1166, sep 2010.

[26] GR Irwin. Analysis of Stresses and Strains Near the End of a Crack Traversing a Plate. J. Appl. Mech., 24:361 - 364, 1957.

[27] SA Khristianovic and YP Zheltov. Formation of vertical fractures by means of highly viscous fluids. In Proc. 4th World Petroleum Congress, Rome, volume 2, pages 579-586, 1955.

[28] Y. Kovalyshen. Fluid-driven fracture in poroelastic medium. $\mathrm{PhD}$ thesis, University of Minnesota, 2010.

[29] B Lecampion. An extended finite element method for hydraulic fracture problems. Commun. Numer. Methods Eng., 25(2):121-133, feb 2009.

[30] S Levasseur, R Charlier, B Frieg, and F Collin. Hydro-mechanical modelling of the excavation damaged zone around an underground excavation at Mont Terri Rock Laboratory. Int. J. Rock Mech. Min. Sci., 47(3):414-425, apr 2010 .

[31] MC Lobao, R Eve, DRJ Owen, and Eduardo A de Souza N. Modelling of hydro-fracture flow in porous media. Eng. Comput., 27(1-2):129-154, 2010 . 
[32] GM Lomize. Water flow through jointed rock. Gosenergoizdat, 1951.

[33] S Mathias and M van Reeuwijk. Hydraulic Fracture Propagation with 3-D Leak-off. Transp. Porous Med., 80:499-518, 2009. 10.1007/s11242009-9375-4.

[34] SL Mitchell, R Kuske, and AP Peirce. An asymptotic framework for finite hydraulic fractures including leak-off. SIAM J. Appl. Math., 67(2):364-386, 2006.

[35] SL Mitchell, R Kuske, and AP Peirce. An asymptotic framework for the analysis of hydraulic fractures: The impermeable case. J. Appl. Mech.-Trans. ASME, 74(2):365-372, mar 2007.

[36] RP Nordgren. Propagation of a vertical hydraulic fracture. Old SPE Journal, 12(4):306-314, 1972.

[37] P Papanastasiou. An efficient algorithm for propagating fluid-driven fractures. Comput. Mech., 24(4):258-267, 1999.

[38] TK Perkins and LR Kern. Widths of hydraulic fractures. J. Pet. Technol., 13(9):937-949, 1961.

[39] E. Sarris and P. Papanastasiou. Modelling of hydraulic fracturing in a poroelastic cohesive formation. Int. J. Geomech., 2001.

[40] E. Sarris and P. Papanastasiou. The influence of the cohesive process zone in hydraulic fracturing modelling. Int. J. Fracture, 167(1):33-45, JAN 2011.

[41] AA Savitski and E Detournay. Propagation of a penny-shaped fluiddriven fracture in an impermeable rock: asymptotic solutions. Int. J. Solids Struct., 39(26):6311-6337, dec 2002.

[42] JM Segura and I Carol. Coupled HM analysis using zero-thickness interface elements with double nodes. Part I: Theoretical model. Int. J. Numer. Anal. Methods Geomech., 32(18):2083-2101, dec 2008.

[43] L Simoni and S Secchi. Cohesive fracture mechanics for a multi-phase porous medium. Eng. Comput., 20(5-6):675-698, 2003.

[44] VC Tsai and JR Rice. A model for turbulent hydraulic fracture and application to crack propagation at glacier beds. J. Geophys. Res., 115(F03007), 2010. 
[45] LM Vandamme and JC Roegiers. Poroelasticity in hydraulic fracturing simulators. J. Pet. Technol., 42(9):1199-1203, SEP 1990.

[46] PA Witherspoon, JSY Wang, K Iwai, and JE Gale. Validity of Cubic Law for fluid flow in a deformable rock fracture. Water Resour. Res., 16(6):1016-1024, 1980.

[47] YP Zheltov and SA Khristianovitch. On the mechanism of hydraulic fracturing of an oil-bearing stratum. Izvest. Akad. Nauk SSR, OTN, 5:3-41, 1955.

[48] G Zimmermann and A Reinicke. Hydraulic stimulation of a deep sandstone reservoir to develop an Enhanced Geothermal System: Laboratory and field experiments. Geothermics, 39(1):70 - 77, 2010. The European I-GET Project: Integrated Geophysical Exploration Technologies for Deep Geothermal Reservoirs. 